Sevilla. Sevilla.

PAU, C. -1921- Diez días en Sierra Morena. Mem. Real Acad. Soc. Esp. Hist. Nat. , 287-298.

PUJADAS, A. -1986- Flora arvense y ruderal de la provincia de Córdoba. Tesis Doctoral. Córdoba.

RIVAS GODAY, S. \& RIVAS MARTINEZ, S. -1968- Matorrales y tomillares de la Península Ibérica. Suplemento n 2. Acerca de los Carthamo-Carduncellus de la Ononido-Rosmarinetea peninsular. Anales Inst. Bot. Cavanilles 25:188-197.

RIVAS MARTINEZ, S. -1975- Sobre la nueva clase Polygono-Poetea annuae. Phytocoenología. 2:123-140.

RUIZ DE CLAVIJO, E. \& CABEZUDO, B. -1979- Aportaciones a la flora de la provincia de Sevilla. Lagascalia 9:39-49.

TUTIN, T.G. -1968- Tordylium, in T.G. TUTIN \& al. (eds.) Flora Europaea 2:367. Cambridge.

WEBB, D.A. -1964- Rumex, in T.G. TUTIN \& al. (eds.) Flora Europaea 1:82-89. Cambridge.

\title{
NOTA SOBRE LA FLORA DE LA SIERRA DE CABO DE GATA (ALMERIA)
}

\author{
F. SANZ FABREGA \& M. COSTA TENORIO \\ Departamento de Botánica y Fisiologia Vegeta1 \\ Facultad de Biología \\ Universidad Complutense de Madrid
}

RESUMEN: Se citan 26 táxones para el piso termomediterráneo de la provincia de Almería. De ellos 5 se mencionan por primera vez para ésta provincia. Otros muchos suponen una ampliación de su área conocida en esta provincia. Se comenta también su situación ecológica.

ABSIRACT: Twenty six thermic mediterranean taxa recorded for Almería. Five of them are mentioned for the first time en this province. Many others suppose an enlargement of their known area. We comment also their ecology.

En esta nota recogemos una serie de táxones de éste territorio seleccionados por su interés, tanto ecológico como corológico. Todo el territorio corresponde al huso $30 \mathrm{~S}$. Los testimonios se encuentran depositados en el Herbario de la Facultad de Biología de la Universidad Complutense (MAC).

El área objeto de estudio se encuentra en la región mediterránea, superprovincia mediterráneo-iberolevantina. Pertenece al séctor almeriense de la provincia murciano-almeriense. Toda ella se encuentra incluida en el piso bioclimático termomediterráneo; el ombroclima oscila entre el semiárido y el árido. Los sustratos son de tipo volcánico con numerosos enclaves de materiales sedimentarios terciarios que ocupan las depresiones internas de las pequeñas sierras. 
Algunos de los táxones que se citan se conocían ya en ésta provincia, pero todas las referencias que hemos encontrado los señalan en pisos bioclimáticos superiores (mesomediterráneo a oromediterráneo) como Euphorbia flavicoma DC., Rumex intermedius DC. in Lam. \& DC., Chaenorhinum origanifolium (L.) Fourr. subsp. crassifolium (Cav.) Rivas Goday \& Borja, Antirrhinum barrelieri Boreau, Ophrys lutea (Gouan) Cav. subsp. lutea. Su presencia en áreas basales, próximas al mar, nos parece resaltable desde un punto de vista ecológico. Otros, son táxones muy pocos citados en la provincia, algunos de los cuales constituyen la primera referencia provincial como Phillyrea media L., Ophrys tenthredinifera Willd. o Datura innoxia Miller, taxon naturalizado de origen centroamericano. Se han incluido otra serie de táxones nitrófilos, ruderales o arvenses, por suponer aportaciones corológicas en la provincia de Almería, aunque se trata de entidades comunes en la Península Ibérica.

Cerastium semidecandrum L. subsp. semidecandrum

Fernán Pérez, cerro Jayón (WF 8587), 350 m, 18-IV-1984, Sanz Fábrega (MAC 17007).

En pastizales terofíticos nitrificados. Poco frecuente, sólo se encuentra en las cotas más altas de este territorio (300-350 m).

Silene vulgaris (Moench) Garcke subsp. commutata (Guss.) Hayek

Fernán Pérez, cerro Jayón (WF 8587), 320 m, 17-IV-1984, Sanz Fábrega (MAC 17000).

Zonas umbrosas en claros de matorral. Primera cita provincial del taxon.

Rumex intermedius DC.

Fernán Pérez, cerro Jayón (WF 8587), 320 m, 13-V-1984, Sanz Fábrega (MAC 17025).

En desmontes, claros de matorral y pedregales subnitrófilos. Señalado por Losa \& Rivas Goday (1968) en zonas más altas, en comunidades seriales de encinar.

Althaea hirsuta L. 17028)

Las Negras, El Aguilón (WF 8785), 340 m, 21-IV-1984, Sanz Fábrega (MAC

Taxon subnitrófilo, en pastizales anuales sobre suelos frescos.

Lepidium graminifolium L. subsp. graminifolium

Rodalquilar (WF 8578), $80 \mathrm{~m}, 13-\mathrm{XI}-1985$, Sanz Fábrega (MAC 17037).

Taxon ruderal y nitrófilo, del que se da a conocer una localidad más basal a las citadas hasta ahora (Losa \& Rivas Goday, 1974).

Mathiola lunata DC.

Entre Los Albaricoques y Rodalquilar (WF 8078), $180 \mathrm{~m}$, 10-IV-1985,

Sanz Fábrega (MAC 17036).

En barbechos y campos eriales. Taxon que Losa \& Rivas Goday (1974)

señalan en zonas mesomediterráneas del interior de la provincia.

Medicago arabica (L.) Hudson

Las Negras, cala de San Pedro (WF 9185), 20 m, 16-III-1984, Sanz Fábrega (MAC 17004).

Pastizaĺes terofíticos sobre suelos muy pedregosos. Taxon del que no conocemos ninguna referencia anterior en la provincia.

Trifolium arvense L.

El Caballón (WF 8792) 200 m, 18-V-1985, Sanz Fábrega (MAC 17006).

Común en pastizales subnitrófilos húmedos. 
Trifolium glomeratum L.

Rodalquilar, barranco de Requena (WF 8278), 220 m, 18-V-1985, Sanz Fábrega (MAC 17046).

Pastizales anuales nitrificados en pequeñas depresiones que acumulan algo de humedad.

Euphorbia flavicoma DC. 17002).

Carboneras, Mesa Roldán (WF 9789), 180 m, 18-V-1985, Sanz Fábrega (MAC

Taxon muy escaso y localizado en esta zona. Ocupa biotopos bastante pedregosos en claros de tomillar. Con esta localidad se amplía notablemente el margen altitudinal de este taxon en la provincia de Almería. Sólo se conocía de las zonas oromediterráneas de la Sierra de Gádor y de la Sierra Nevada almeriense (Losa \& Rivas Goday, 1968; Mota \& Valle, 1985).

Geranium purpureum Vill.

Carboneras, Mesa Roldán (WF 9789), 200 m, 20-III-1984, Sanz Fábrega, (MAC 17043).

Pastizales escionitrófilos en pedreras y canchales, sobre suelos húmedos y frescos.

Ammoides pusilla (Brot.) Breistr. Fernán Pérez, cerro Jayón (WF 8587), 3ุ20 m, 15-V-1985, Sanz Fábrega (MAC 17010). Común en pastizales anuales algo húmedos y nitrificados.

Bupleurum fruticescens L.

Rodalquilar, cerro de El Romeral (WF 8879), 180 m, 3-VII-1985, Sanz Fábrega (MAC 17033).

Poco frecuente, en matorrales sobre sustrato calcáreo y suelos poco profundos.

Smyrnium olusatrum L.

Fernán Pérez, cerro Jayón (WF 8587), 320 m, 2-V-1984, Sanz Fábrega

(MAC 17016).

En comunidades escionitrófilas de cunetas y bordes de camino.

Datura innoxia Miller

Los Escullos (WF 7284), 20 m, 25-VIII-1985, Sanz Fábrega (MAC 17021).

Taxon ruderal, poco común. De origen centroamericano y naturalizado en esta zona. No conocemos ninguna referencia del mismo en la provincia de Almería.

Phillyrea media L.

Rodalquilar, La Rellana (WF 7781), 450 m, 4-III-1985, Sanz Fábrega (MAC 17047).

Primera cita provincial de este taxon. En matorrales de Phlomidion almeriensis, muy localizado junto con Cistus ladanifer, sobre rocas impermeables.

Antirrhinum barrelieri Boreau 17009)

Las Negras, San Pedro (WF 9084), 220 m, 14-V-1984, Sanz Fábrega (MAC

Taxon muy localizado en esta zona, en repisas arenoso-limosas. Citado anteriormente en Sierra Nevada (Alcaraz \& col., 1981).

Chaenorhinum origanifolium (L.) Fourr. subsp. crassifolium (Cav.) Rivas Goday \& Borja

San José, Los Escullos (WF 8568), 10 m, 10-III-1985, Sanz Fábrega (MAC 17038). 
En rocas calizas, con Sarcocapnos enneaphylla. Hasta el momento tan solo se conocía en la provincia, de Sierra Nevada (Mota \& Valle, 1985).

Crupina vulgaris Cass.

Carboneras, el Cabalión (WF 8791), 250 m, 18-V-1985, Sanz Fábrega (MAC $17040)$.

Poco frecuente, en pastizales nitrificados sobre suelos arenosos.

Echinops strigosus L. 17041).

Agua Amarga, El Plomo (WF 9386), 2 m, 7-VII-1985, Sanz Fábrega (MAC

En comunidades nitrófilas de bordes de caminos.

Xanthium spinosum L. 17048).

Agua Amarga, El Plomo (WF 9386), 10 m, 3-XI-1984, Sanz Fábrega (MAC

Taxon naturalizado, nitrófilo y arvense.

Carex extensa Good.

Barranco de Las Negras (WF 8782), 20 m, 23-V-1985, Sanz Fábrega (MAC 17020).

Poco común en medios húmedos, junto a arroyos constantes casi todo el año. Taxon muy poco citado en la provincia (Alcaraz \& Garre, 1984, Morales $\& \operatorname{col} ., 1983)$.

Carex hallerana Asso

Carboneras, Rellana de las Contraviesas (WF 9592), $120 \mathrm{~m}, 21-\mathrm{V}-1985$, Sanz Fábrega (MAC 17030).

Aparece, aunque escasa, en claros de tomillar sobre roca caliza. Losa \& Rivas Goday (1968) la mencionan en los pisos meso y supramediterráneos.

Barlia robertiana (Loisel.) W. Greuter

Rodalquilar, barranco de Rẹuena (WF 8278), 180 m, 4-III-1984, Sanz Fábrega (MAC 17019).

En pastizales umbrosos, sobre suelos profundos. Sólo conocemos una cita anterior de este taxon en la provincia de Almería.

Ophrys lutea (Gouan) Cav. subsp. lutea

Fernán Pérez, cerro Jayón (WF 8587), 320 m, 18-IV-1984, Sanz Fábrega (MAC 17034).

Zonas frescas entre matorral. Añadimos otra localidad de éste taxon conocido solamente de zonas más altas (Losa \& Rivas Goday, 1974).

Ophrys tenthredinifera Willd.

Fernán Pérez, cerro de La Higuera (WF 8886), 320 m, 21-III-1984, Sanz Fábrega (MAC 17032).

Lugares umbrosos y protegidos entre matorral. Primera cita provincial del taxon.

\section{BIBLIOGRAFIA}

ALCARAZ, F. \& col. -1981- Aportación al conocimiento de la flora cormofítica de Almería. Anal. Univ. Murcia Cienc. 37(1-4):1-37.

ALCARAZ, F. \& GARRE, M. -1984- Notas sobre la flora del sureste Ibérico. III. Collect. Bot., 15:13-16.

LOSA, M.T. \& RIVAS GODAY, S. -1968-1974- Estudio florístico y geobotánico 
de la provincia de Almeria. Arch. Inst. Aclim. C.S.I.C. 13(I):I-III; $13(2):$ II2-237.

MORALES, C., SANCHEZ-CASTILLO, P. \& ROMERO-GARCIA, A.T. -1983- Notas florísticas y corológicas de Andalucía Oriental. Lazaroa 5:215-219.

MOTA, J.F. \& VALLE, F. -1985- Notas corológicas sobre la flora de la provincia de Almería (España). Candollea 40:563-570.

\section{CIANOFICEAS INTERESANTES DE LA CUENCA DEL RIO SEGURA (SE DE ESPAÑA)}

M. ABOAL ( $\because)$ \& X. LLIMONA (㚇)

Dpto. de Botánica. Facultad de Biologia.

(*) Universidad de Murcia

(

RESUMEN: Se aportan citas de dos cianófitas, hasta ahora desconocidas en nuestro país: Capsosira viride (Frèmy) Bourelly y Homeothrix articulata Starmach.

SUMMARY: Two new records for the Sapnish epicontinental waters are reported: Capsosira viride (Frèmy) Bourelly and Homeothrix articulata Starmach.

Dentro del estudio que estamos realizando de la flora algal de la Cuenca del Río Segura, aportamos el hallazgo de dos especies de Cianofíceas, que representan dos nuevas citas para la flora algal española, en base a la bibliografía consultada.

Capsosira viride (Frèmy) Bourelly

Caracterizada por su talo pulviniforme, gelatinoso, pardoamarillento. Filamentos de 7-9 m de diámetro. Vainas hialinas o amarillentas, lisas. Células cuadrangulares o ligeramente más largas que anchas, de 5-6 x 5-8 $\mathrm{m}$. Heterocistes intercalares muy escasos. Planococos formados en las células apicales.

Murcia. Rambla del Moro. Cieza. XH 4332 (IX-83).

Los talos amarillentos o parduzcos ocupan las hendiduras de las rocas del : fondo de cursos de agua alcalina salobre (Tabla 1), de escasa profundidad y con fuerte insolación.

Esta especie es propia de países cálidos; ha sido citada de Africa (Geitler \& Ruttner, 1935) y de la India (Desikachary, 1959). En estos trabajos se indica su preferencia por sistemas lóticos pero no se hace mención de la calidad físico-química del agua.

Homeothrix articulata Starmach

Filamentos solitarios o en pequeños grupos, de $5 \mathrm{~m}$ de diámetro en la base, progresivamente atenuados hasta formar el pelo terminal; generalmente erectos. Alcanzan $460 \mathrm{~m}$ de longitud. Vainas hialinas no estratificadas. Tricomas constrictos en los tabiques, verdeazulados, de 4,5-5 $\mathrm{m}$ de diámetro. Células isodiométricas o deprimidas. 